expression and knockdown of these factors in the tumour reduced tracheal branches and tumour size and increased fly survival. Prevention of ROS production in the tumour reduced tracheal coverage, ISC mitosis, and tumour growth. However, it remains difficult to dissociate a ROS function on vascularization of the tumour from previously described roles of ROS directly on stem cell proliferation ${ }^{14,15}$. Nevertheless, the conservation of mechanisms and factors involved in damage response and upon tumorigenesis in the fly intestine and neo-angiogenesis is evident.

An interesting finding reported by both groups is the reversibility of tracheogenesis after epithelial damage ${ }^{5}$ or upon tumorigenesis ${ }^{6}$ (after switching off Ras ${ }^{\mathrm{V} 12}$ expression). Thus, a future aim for the field will be to better understand mechanisms and factors regulating trachea plasticity during this recovery phase concomitant with the slowdown of stem cell proliferation.

In summary, both studies establish the Drosophila intestine as an excellent example of tissue collaboration regulating adult stem cells, tissue regeneration or tumour growth. Future studies using this model will provide a better understanding of the mechanisms required for co-regulation and dynamic plasticity of vascular tissues and stem cell responses.

Louis Gervais ${ }^{\bowtie}$ and Allison J. Bardin (D) Institut Curie, PSL Research University, CNRS UMR 3215, INSERM U934, Stem Cells and Tissue Homeostasis Group, Paris, France.

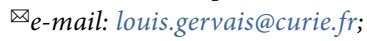
allison.bardin@curie.fr

Published online: 26 May 2021

https://doi.org/10.1038/s41556-021-00695-w
References

1. Ding, L., Saunders, T. L., Enikolopov, G. \& Morrison, S. J. Nature 481, 457-462 (2012).

2. Kiel, M. J. et al. Cell 121, 1109-1121 (2005).

3. Shen, Q. et al. Cell Stem Cell 3, 289-300 (2008)

4. De Palma, M., Biziato, D. \& Petrova, T. V. Nat. Rev. Cancer 17, 457-474 (2017).

5. Perochon, J. et al. Nat. Cell Biol. https://doi.org/10.1038/s41556021-00676-z (2021).

6. Tamamouna, V. et al. Nat. Cell Biol. https://doi.org/10.1038/ s41556-021-00674-1 (2021).

7. Gervais, L. \& Bardin, A. J. Curr. Opin. Cell Biol. 48, 97-105 (2017).

8. Li, Z., Zhang, Y., Han, L., Shi, L. \& Lin, X. Dev. Cell 24 133-143 (2013).

9. Linneweber, G. A. et al. Cell 156, 69-83 (2014).

10. Hayashi, S. \& Kondo, T. Genetics 209, 367-380 (2018).

11. Centanin, L. et al. Dev. Cell 14, 547-558 (2008)

12. Fraisl, P., Mazzone, M., Schmidt, T. \& Carmeliet, P. Dev. Cell 16, 167-179 (2009).

13. Campbell, K. et al. Nat. Commun. 10, 2311 (2019).

14. Hochmuth, C. E., Biteau, B., Bohmann, D. \& Jasper, H. Cell Stem Cell 8, 188-199 (2011).

15. Patel, P. H. et al. Nat. Commun. 10, 4365 (2019).

\title{
Immune memory in individuals with COVID-19
}

\author{
COVID-19 has led to a global pandemic, but the long-term immunological effects of the infection are only partially \\ understood. A new study now provides important new clues by describing the transcriptional and epigenetic \\ processes behind the immune memory of both adaptive and innate immune cells in individuals who have recovered \\ from COVID-19.
}

\section{Mihai G. Netea and Yang Li}

nitially emerging in China in December 2019, the coronavirus disease COVID-19 caused by severe acute respiratory syndrome coronavirus-2 (SARS-CoV-2) has become a major health crisis, with high morbidity and mortality, especially among the elderly and people with various comorbidities ${ }^{1}$. Much has been learned in the past year regarding pathophysiology of the disease, in which immune-based mechanisms play a major role not only for protection, but also for immunopathology. While novel therapies have been developed based on immunomodulatory drugs (such as dexamethasone or IL-6 blockers), vaccination remains the most effective approach to blocking the spread of the virus and alleviate the consequences of the pandemic.

Several successful vaccines have been already developed based on various technological platforms, including mRNA technology, adenovirus platforms, recombinant proteins or inactivated viruses. While several of these have proved to be effective in short-term phase 3 trials $^{2}$, little is known regarding the duration of their effects and the precise immune correlates of protection mediating the protection. It is therefore crucial to understand in detail the immunological memory processes inducing protection against the virus after natural COVID-19 infection. We would be thus able to employ more rationally the available vaccines and even design and develop the next generation of vaccines with improved and longer protection against infection. In this issue of Nature Cell Biology, You et al. now make one important step in that direction by describing the transcriptional and epigenetic processes behind the long-term memory of immune cells in individuals who have recovered from COVID- $19^{3}$. By using cutting-edge single-cell sequencing technology, the authors describe the transcriptional modules, the regulatory nodes at the level of transcription factors, and the chromatin accessibility in various immune cell types after COVID-19 recovery.

The first line of evidence provided by You and colleagues addresses the mechanisms of adaptive immune memory at the level of B cells and T cells (Fig. 1). Lymphocytes are crucial components of the long-term protection induced either by natural infection or by vaccination; while their role as correlates of protection against COVID-19 has not been formally demonstrated, it is widely assumed that they mediate the protection induced by natural infection or vaccines ${ }^{4,5}$. First, You et al. identified important differences in the developmental processes in B lymphocytes from COVID19 convalescent individuals compared to healthy volunteers, based on differential B-cell lineage trajectories in chromatin accessibility. Second, the authors also identified crucial differences in transcription factor (TF) regulators, with the NF- $\kappa \mathrm{B}$ subunits RELA and RELB being enriched in B cells of healthy volunteers, while 


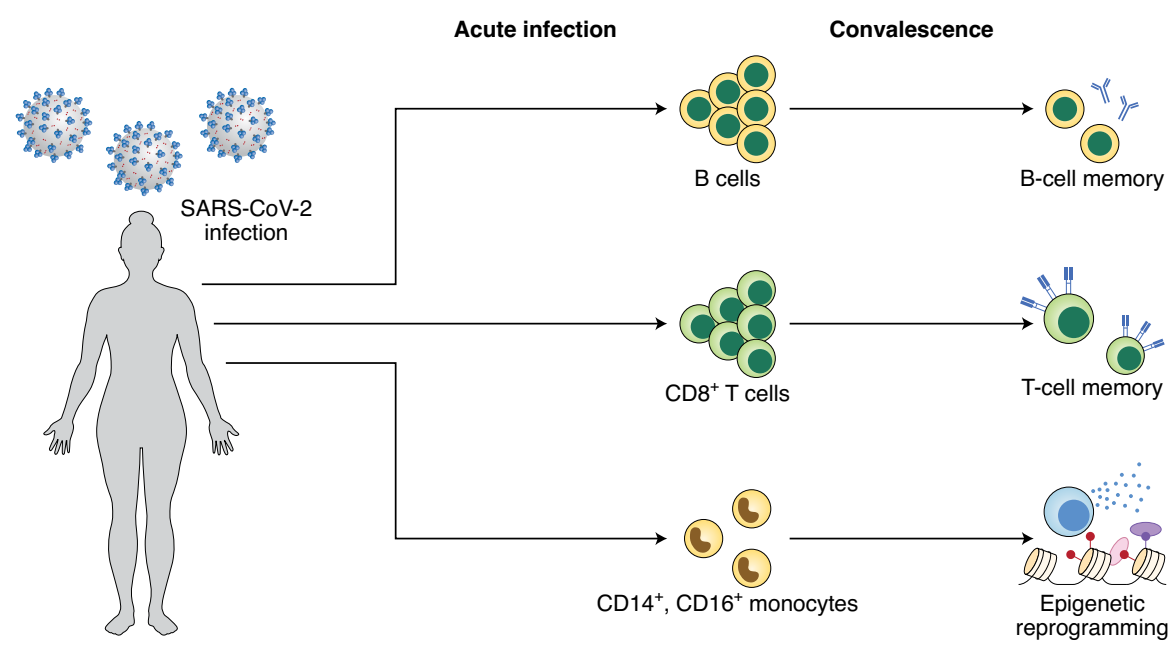

Fig. 1 | Memory responses after COVID-19 infection. COVID-19 infection leads to proliferation of B cells and T cells, which is followed by the survival of a few memory $B$ and $T$ cells that insure long-term protection after elimination of the virus. COVID-19 infection also leads to potent activation of myeloid cells such as monocytes and macrophages. Interestingly, recovery from the infection is accompanied by long-term transcriptional, epigenetic and functional rewiring of monocytes (also called trained immunity), inducing changes in the innate immune responses after COVID-19.

activator protein $1(\mathrm{AP}-1)$ transcription factors FOS and JUN being higher in naïve, memory and plasma B cells of COVID-19 convalescent individuals. Based on the integrated timing of TF activity, the authors concluded that TFs expressed in B cells of healthy individuals promote maintenance and homeostasis, while inducing B-cell activation, differentiation, and IgG class switch recombination in COVID-19 convalescent individuals. Third, You and colleagues described the transcriptional and chromatin landscape of T cells after infection with SARS-CoV-2. In contrast to healthy volunteers, COVID-19 convalescent individuals displayed enhanced effector and memory $\mathrm{CD}^{+}$cells, which is not surprising considering their role in antiviral immunity. The authors also described the TF regulators in $\mathrm{CD}^{+}$cells, which again comprise the AP-1 factors FOS and JUNB, supported by earlier literature ${ }^{6}$. Memory commitment on the other hand involves Kruppel-Like Factors 2 and 3. Interestingly, T-cell receptor clonality analysis showed a very substantial expansion of $\mathrm{CD}^{+}$ clones, while $\mathrm{CD} 4^{+} \mathrm{T}$ cells displayed diverse repertoire with minimal expansion. Altogether, the transcriptional and epigenetic analysis of lymphocytes from COVID-19 convalescent individuals argues for an important role of $\mathrm{B}$ cells and $\mathrm{CD}^{+}$ $\mathrm{T}$ cells in mediating memory responses after natural infection, and it is rational to explore these pathways for improvement of vaccination efficacy.
While memory responses in B and $\mathrm{T}$ lymphocytes are expected after a viral infection, a more surprising finding of You and colleagues relates to the identification of long-term adaptive changes in monocytes as well. Adaptive characteristics in the myeloid cell lineage have been recently described after certain infections, and especially vaccinations, resulting in improved responses (cytokine and ROS production, phagocytosis, microbial killing) of innate immune cells upon stimulation with heterologous stimuli. This de facto non-specific immunological memory of myeloid cells is mediated by transcriptional and epigenetic rewiring, and has been termed 'trained immunity" Interestingly, the authors identified a trained immunity phenotypic program induced by SARS-CoV-2 infection in both CD14 ${ }^{+}$ and $\mathrm{CD} 16^{+}$monocytes from COVID-19 convalescent individuals (Fig. 1). This program included increased chromatin accessibility in monocytes after recovery from COVID-19 for both IL1B and chemokine genes, which are well known to be both important for trained immunity ${ }^{8}$ and strongly activated in COVID-19. Increased chromatin accessibility was associated with higher production of these cytokines after stimulation, further confirming a trained phenotype in monocytes in post-COVID19 individuals.

The identification of trained monocytes after recovery from SARS-CoV-2 is important at several levels. On one hand, this provides a more comprehensive understanding of the changes in immune responses after infection with COVID-19, demonstrating long-term adaptation not only in lymphocytes but also in myeloid cells. On the other hand, the discovery of these processes is also exciting for understanding the potential consequences of the disease. It is tempting to speculate that induction of trained immunity could contribute to the long-term protection against reinfection, and that this needs to be considered for the development of future vaccines. However, such enhanced responsiveness of myeloid cells may lead in some individuals to adverse hyperactivation, such as in multisystem inflammatory syndromes in adults (MIS-A) or children (MIS-C), or even in people with long-term symptoms after COVID ${ }^{10}$.

All in all, the study describes for the first time important regulatory nodes of memory in adaptive immunity and opens the door to a new field in COVID-19 research showing long term-regulation and trained immunity in myeloid cells of convalescent individuals. Future studies are warranted to explore these processes in more depth, and larger studies are needed to assess potential differences between patients with mild, moderate, severe COVID-19 or convalescent individuals. Systems approaches based on multi-omics data will be necessary to reveal the underlying molecular circuits of immune response to viral infection, longitudinal studies will be needed to understand the kinetics of these processes, and clinical and translational studies should be done to understand the potential use of this knowledge for the treatment of late complications of COVID-19, and even improve vaccination. Only with such future studies the potential of these discoveries will be fulfilled.

Mihai G. Netea (D) 1,2四 and Yang Li ${ }^{1,3,4}$ ${ }^{1}$ Department of Internal Medicine and Radboud Center for Infectious diseases (RCI), Radboud University Nijmegen Medical Centre, Nijmegen, the Netherlands. ${ }^{2}$ Department for Genomics \& Immunoregulation, Life and Medical Sciences Institute (LIMES), University of Bonn, Bonn, Germany. ${ }^{3}$ Department of Computational Biology for Individualised Infection Medicine, Centre for Individualised Infection Medicine (CiiM), Helmholtz-Centre for Infection Research (HZI) and the Hannover Medical School (MHH), Hannover, Germany. ${ }^{4}$ TWINCORE, Centre for Experimental and Clinical Infection Research, Helmholtz-Centre for Infection Research (HZI) and the Hannover Medical School (MHH), Hannover, Germany.

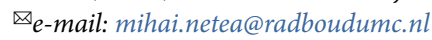


Published online: 9 June 2021

https://doi.org/10.1038/s41556-021-00689-8

References

1. Wolff, D., Nee, S., Hickey, N. S. \& Marschollek, M. Infection 49, 15-28 (2021).

2. Kim, J. H., Marks, F. \& Clemens, J. D. Nat. Med. 27, 205-211 (2021).

3. You, M. et al. Nat. Cell Biol. https://doi.org/10.1038/s41556-02100690-1 (2021).
4. Mulligan, M. J. et al. Nature 586, 589-593 (2020).

5. Anderson, E. J. et al. N. Engl. J. Med. 383, 2427-2438 (2020).

6. Satpathy, A. T. et al. Nat. Biotechnol. 37, 925-936 (2019).

7. Netea, M. G. et al. Nat. Rev. Immunol. 20, 375-388 (2020).

8. Arts, R. J. W. et al. Cell Host Microbe 23, 89-100 e5 (2018).

9. Giamarellos-Bourboulis, E. J. et al. Cell Host Microbe 27, 992-1000.e3 (2020).

10. Vogel, T. P. et al. Vaccine https://doi.org/10.1016/j.vaccine. 2021.01.054 (2021).
Acknowledgements

M.G.N. was supported by an ERC Advanced Grant (\#833247) and a Spinoza Grant from the Netherlands Organization for Scientific Research. Y.L. was supported by an ERC Starting Grant (\#948207) and the Radboud

University Medical Centre Hypatia Grant (2018).

Competing interests

The authors declare no competing interests.

\title{
The ELOF(1)ant in the room of TCR
}

\author{
Transcription-coupled repair of DNA lesions takes place across all domains of life. A transcription-elongation \\ factor, ELOF1, highly conserved in eukaryotes, has now been shown to play a key role in this process. Importantly, \\ these findings also include evidence of a second transcription-coupled repair pathway in mammalian cells.
}

\section{Christopher J. Carnie and Stephen P. Jackson}

T he repair of DNA lesions-such as those caused by ultraviolet (UV) light-is critical for cell function and survival. Transcription-coupled repair (TCR) of such lesions is conserved from bacteria to humans, with mutations in the constituent genes of the TCR pathway underlying several human diseases ${ }^{1}$. In this issue of Nature Cell Biology, Geijer et al. and van der Weegen et al. identify a central role for an RNA Polymerase II (RNAPII) transcription-elongation factor, ELOF1, in TCR and propose an elegant mechanism by which ELOF1 regulates a key step in this process. Furthermore, these two papers provide evidence for a previously unappreciated mammalian TCR pathway ${ }^{2,3}$.

DNA lesions caused by UV light are some of the most common forms of DNA damage and can have drastic consequences for human health. As such, understanding the types of UV-induced DNA lesions and their mechanisms of repair is of great interest to researchers and clinicians. UV-induced DNA perturbations, such as cyclobutane pyrimidine dimers (CPDs), are bulky lesions that can impede transcription and are repaired by a process termed nucleotide excision repair (NER). NER has two known subpathways that differ based on the mode of lesion sensing: global genome-NER (GG-NER) and transcription-coupled-NER (TC-NER). While GG-NER can repair UV lesions anywhere in the genome, lesions that occur on the transcribed strand of gene bodies can be repaired much more quickly by TC-NER (Fig. 1) ${ }^{1}$. NER's importance is underlined by the range of human diseases caused by loss-of-function mutations in NER genes, including Cockayne syndrome (caused by mutations in CSA or CSB), UV sensitivity syndrome (caused by mutations in UVSSA), and xeroderma pigmentosum (caused by mutations in the XPA-G genes) ${ }^{1}$.

Notably, TC-NER of transcription-blocking lesions (TBLs) is initiated by the transcription machinery itself. Elongating RNAPII, upon stalling at a TBL, acts as both an apical damage sensor and a recruitment platform for TC-NER factors. Stalled RNAPII is recognised by the protein CSB, which binds RNAPII 3' to the lesion and uses its $3^{\prime}-5^{\prime}$ translocase activity to try to 'push' RNAPII past the lesion. While smaller lesions might be bypassed, bulkier lesions such as CPDs remain insurmountable by CSB activity. In this case, the CRL4 $4^{\mathrm{CSA}}$ complex is recruited, consisting of CSA, DDB1 and the CUL4A-RBX1 E3 ubiquitin ligase ${ }^{1}$. CRL4 $4^{\mathrm{CSA}}$ stimulates Cullin-dependent ubiquitylation of RNAPII's largest subunit, RPB1 (RNA polymerase II subunit $\mathrm{B} 1$ ), at lysine residue 1268 , which supports the recruitment to and stabilisation of mono-ubiquitylated UVSSA at the damage site ${ }^{4,5}$.

Stabilisation of mono-ubiquitylated UVSSA at the damage site represents a critical step in TC-NER. At this stage, RNAPII must be moved back from the lesion to allow downstream repair factors to access the damage. To facilitate this, UVSSA recruits the transcription initiation and repair complex transcription factor IIH (TFIIH), which through the reverse $\left(5^{\prime}-\right.$ $\left.3^{\prime}\right)$ translocase activity of its XPD subunit, moves along the DNA until it is blocked by the lesion. This helps dislodge the stalled RNAPII, enabling lesion licensing by TFIIH and XPA. The TFIIH-XPA complex then positions the endonucleases ERCC1-XPF and XPG at either side of the lesion. ERCC1-XPF and XPG perform a 'dual incision' step, excising a stretch of DNA 22-30 nucleotides long containing the lesion. The resultant single-stranded DNA gap is then repaired by gap-filling DNA synthesis by DNA polymerase $\delta, \varepsilon$ or $\kappa$ using the undamaged strand as a template ${ }^{1}$.

ELOF1 was recently proposed as a candidate TC-NER factor following extensive genome-wide CRISPR-Cas9 screens using genotoxic insults including $\mathrm{UV}$ and Illudin $\mathrm{S}^{6}$, an alkylating agent that causes damage thought to be repaired almost exclusively by TC-NER. Geijer et al. and van der Weegen et al. identified ELOF1 as a gene whose knockout sensitises cells to UV and Illudin S, respectively, in their CRISPR-Cas9 screens ${ }^{2,3}$. After validating these phenotypes, the authors demonstrate that following UV-induced transcription arrest, $E L O F 1^{-/-}$cells display impaired transcription recovery compared to wild-type (WT) cells, a classic hallmark of a TC-NER defect ${ }^{2,3}$. Furthermore, through a ChIP-seq-based method termed 'TCR-seq', van der Weegen et al. find that UV-induced DNA damage persists on the transcribed strand of ELOF1 $1^{-1-}$ cells ${ }^{3}$. Notably, both papers demonstrate that ELOF1 acts as a transcription-elongation factor in unperturbed conditions and interacts constitutively with the transcription 